\title{
Properties of polylactic acid composites reinforced with oil palm biomass microcrystalline cellulose
}

\begin{abstract}
In this work, polylactic acid (PLA) composites filled with microcrystalline cellulose (MCC) from oil palm biomass were successfully prepared through solution casting. Fourier transform infrared (FT-IR) spectroscopy indicates that there are no significant changes in the peak positions, suggesting that incorporation of MCC in PLA did not result in any significant change in chemical structure of PLA. Thermogravimetric analysis was conducted on the samples. The T50 decomposition temperature improved with addition of MCC, showing increase in thermal stability of the composites. The synthesized composites were characterized in terms of tensile properties. The Young's modulus increased by about 30\%, while the tensile strength and elongation at break for composites decreased with addition of MCC. Scanning electron microscopy (SEM) of the composites fractured surface shows that the MCC remained as aggregates of crystalline cellulose. Atomic force microscopy (AFM) topographic image of the composite surfaces show clustering of MCC with uneven distribution.
\end{abstract}

Keyword: Microcrystalline cellulose; Thermal properties; Atomic force microscopy; Casting. 